\title{
An irritable bowel syndrome subtype defined by species-specific alterations in faecal microbiota
}

\author{
Jeffery, I. B., O' Toole, P. W., Ohman, L., Claesson, M. J., Deane, J., Quigley, E. M. M., \& Simren, M. \\ (Accepted/In press). An irritable bowel syndrome subtype defined by species-specific alterations in faecal \\ microbiota. GUT, 61, 997-1006. https://doi.org/10.1136/gutjnl-2011-301501
}

Link to publication record in Ulster University Research Portal

\author{
Published in: \\ GUT
}

\section{Publication Status:}

Accepted/In press: 22/10/2011

DOI:

10.1136/gutjnl-2011-301501

\section{Document Version}

Publisher's PDF, also known as Version of record

\section{General rights}

Copyright for the publications made accessible via Ulster University's Research Portal is retained by the author(s) and / or other copyright owners and it is a condition of accessing these publications that users recognise and abide by the legal requirements associated with these rights.

\section{Take down policy}

The Research Portal is Ulster University's institutional repository that provides access to Ulster's research outputs. Every effort has been made to ensure that content in the Research Portal does not infringe any person's rights, or applicable UK laws. If you discover content in the Research Portal that you believe breaches copyright or violates any law, please contact pure-support@ulster.ac.uk. 
- Additional materials are published online only. To view these files please visit the journal online (http://gut.bmj. com/content/61/7.toc).

${ }^{1}$ Department of Microbiology, University College Cork, Cork, Ireland

${ }^{2}$ Alimentary Pharmabiotic Centre, University College Cork, Cork, Ireland

${ }^{3}$ Department of Internal

Medicine, Institute of Medicine, Sahlgrenska Academy,

University of Gothenburg,

Gothenburg, Sweden

\section{Correspondence to}

Dr Paul W O'Toole, Department of Microbiology, University

College Cork, 447 Food Science

Building, Cork, Ireland

pwotoole@ucc.ie

Accepted 22 October 2011

Published Online First

16 December 2011

\title{
An irritable bowel syndrome subtype defined by species-specific alterations in faecal microbiota
}

\author{
Ian B Jeffery, ${ }^{1}$ Paul W O'Toole, ${ }^{1,2}$ Lena Öhman, ${ }^{3}$ Marcus J Claesson, ${ }^{1,2}$ \\ Jennifer Deane, ${ }^{1,2}$ Eamonn M M Quigley, ${ }^{2}$ Magnus Simrén ${ }^{3}$
}

\section{ABSTRACT}

Background and aims Irritable bowel syndrome (IBS) is a common functional gastrointestinal disorder that may be triggered by enteric pathogens and has also been linked to alterations in the microbiota and the host immune response. The authors performed a detailed analysis of the faecal microbiota in IBS and control subjects and correlated the findings with key clinical and physiological parameters.

Design The authors used pyrosequencing to determine faecal microbiota composition in 37 IBS patients (mean age 37 years; 26 female subjects; 15 diarrhoeapredominant IBS, 10 constipation-predominant IBS and 12 alternating-type (BS) and 20 age- and gendermatched controls. Gastrointestinal and psychological symptom severity and quality of life were evaluated with validated questionnaires and colonic transit time and rectal sensitivity were measured.

Results Associations detected between microbiota composition and clinical or physiological phenotypes included microbial signatures associated with colonic transit and levels of clinically significant depression in the disease. Clustering by microbiota composition revealed subgroups of IBS patients, one of which $(n=15)$ showed normal-like microbiota composition compared with healthy controls. The other IBS samples ( $n=22)$ were defined by large microbiota-wide changes characterised by an increase of Firmicutes-associated taxa and a depletion of Bacteroidetes-related taxa.

Conclusions Detailed microbiota analysis of a wellcharacterised cohort of IBS patients identified several clear associations with clinical data and a distinct subset of IBS patients with alterations in their microbiota that did not correspond to IBS subtypes, as defined by the Rome II criteria.

\section{INTRODUCTION}

Irritable bowel syndrome (IBS) is a common gastrointestinal (GI) disorder that affects 10-20\% of adults in industrialised countries. ${ }^{1}$ Although it has a non-fatal prognosis, IBS patients report more ailments than the general population and commonly incur a diminution in quality of life. ${ }^{1}$ Characteristic symptoms include abdominal pain and/or discomfort, bloating, distension and altered bowel habits. ${ }^{1}$

No single unifying cause has been identified for IBS, but there is recent evidence suggesting the involvement of the gut microbiota. The clearest evidence is provided by subjects who develop IBS de novo following an episode of enteric

\section{Significance of this study}

What is already known on this subject?

- Irritable bowel syndrome (IBS) onset may follow enteric infections.

- A low-grade inflammatory response has been described in IBS.

- Various changes in the microbiota have been described in IBS but their primacy has not been defined.

\section{What are the new findings?}

- A detailed assessment of the faecal microbiota in IBS does not reveal a uniform change in the microbiota.

- Analysis of microbial populations in IBS reveals distinct clusters, of which some overlap with normal controls while others are quite different.

- An increased Firmicutes:Bacteroidetes ratio best characterises those IBS subjects who differ from normal populations.

- The microbial signature is related to the clinical phenotype in a subset of IBS patients.

How might it impact on clinical practice in the foreseeable future?

- Microbial fingerprinting may help to identify IBS subpopulations with differing prognosis and varied therapeutic responses.

infection-postinfectious IBS. Postinfectious IBS has been associated with a number of bacterial species. $^{2-4}$ This and the hypothesis that small intestinal bacterial overgrowth might also contribute to IBS symptomatology $y^{5}$ have motivated investigation of antibiotics as therapeutic agents in IBS. ${ }^{6}$ Large double-blind studies of some antibiotics have shown an improvement (albeit modest, though statistically significant) in IBS symptoms. The improvement has been attributed to a reduction in total bacterial load and, perhaps, an associated suppression of certain causative bacterial species. $^{6}$

The gut microbiota of IBS subjects has thus attracted considerable interest, and alterations in microbiota composition have been linked to IBS and IBS-related symptoms. To cope with the heterogeneity in IBS symptoms, ${ }^{8}$ these studies often employ the Rome criteria to identify IBS subtypes (reviewed by Salonen et al). ${ }^{9}$ Their meta-analysis of IBS microbiota, as determined by 
high-throughput methods, identified consistent microbiota alterations associated with fluctuations in Firmicutes-associated taxa; alterations in the proportions of the Bacteroidetes and Proteobacteria phyla also featured in several studies. ${ }^{10-15}$ However, in studies to date, the phenotypic characterisation of the subjects has often been suboptimal. For example, links to symptom pattern and severity have not been clearly established. Moreover, it is currently unclear if the gut microbiota alterations found were characteristic of IBS patients in general or only to subgroups thereof.

The aim of this study was, therefore, to identify differences in the intestinal microbiota between healthy and IBS subjects in a very well phenotyped cohort. Using faeces as a proxy for the distal bowel, we sought to identify phylum, genus and species level bacterial signatures of IBS. We further aimed to identify operational taxonomic units (OTUs) associated with IBS and their relationship to clinical patterns and physiological measures.

\section{METHODS}

\section{Recruitment of subjects}

IBS patients fulfilling Rome II criteria ${ }^{16}$ were recruited from the Gastroenterology Clinic, Sahlgrenska University Hospital, Gothenburg, Sweden. The samples were collected between January and December 2004 to cover a complete calendar year and eliminate seasonal effects. All patients underwent appropriate diagnostic tests to rule out organic GI disorders as described in the online supplementary methods. Patients with other GI conditions explaining their symptoms, or with another severe coexisting disease, were excluded. Of the 37 patients, 27 were on no medication during the study; 3 were on a proton pump inhibitor, 2 used spasmolytics, 4 bulking agents, 4 selective serotonin reuptake inhibitors, 2 low dose tricyclics and 2 loperamide. We also recruited a group of healthy volunteers without previous or current GI symptoms or history of a chronic disease. These subjects were recruited through local advertisement and completed a GI symptom questionnaire to ensure the absence of current GI symptoms. Antibiotic treatment within the month before stool collection was an exclusion criterion. Subjects were not controlled for probiotic, prebiotic or synbiotic use. The study was approved by the Regional Ethical Review Board at the University of Gothenburg.

Patients who enrolled in the study completed the following questionnaires to assess their GI and psychological symptoms, as well as general quality of life (see online supplementary methods): the Hospital Anxiety and Depression (HAD) Scale ${ }^{17}$; Gastrointestinal Symptom Rating Scale-IBS ${ }^{18}$; Bristol Stool Form Scale-1 week diary to assess stool form ${ }^{19}$; and the Short Form-36 $\left(\right.$ SF-36). ${ }^{20}$ Some subjects $(n=36)$ also underwent a rectal sensitivity test with a rectal barostat ${ }^{21}$ and colonic transit time measurement using radiopaque markers $(n=28)^{22}$ (see online supplementary methods).

\section{Statistical and clustering analysis}

We sequenced approximately 30000 16S rRNA gene V4 region amplicons per subject (see online supplementary methods), and determined the corresponding microbiota composition using established bioinformatics processes. ${ }^{23}$ In brief, quality filtering was applied using the Oiime settings of no ambiguous bases, a mean quality score above 25 , a mean window quality score above 25 , a maximum homopolymer run not exceeding a limit of six and no mismatches in the primer. Similar sequences were clustered at a $97 \%$ sequence identity into OTUs, which can be treated as sequence-based bacterial divisions. The reads were assigned to taxonomies using the Ribosomal Database Project (RDP) classifier. ${ }^{24}$ A confidence value of 0.5 was considered a positive identification. At each phylogenetic classification level, the overall data from each subject was normalised by scaling to an intensity of 1 in order to control for differing numbers of reads. To visualise the data, we employed principal coordinates analyses (PCoAs) based upon the Unifrac distances ${ }^{25}$; the UniFrac measurement indicates the extent to which microbial communities share branch length, based on a phylogenetic tree of all the amplicon sequences in the combined dataset. Secondary clustering analysis was carried out on the OTU abundances by adding 0.000001 to remove zeros followed by dual scaling a log transformed OTU dataset to limits of $-3,3$ and using unsupervised hierarchical cluster analysis with the Pearson correlation coefficient and average linkage. All sequence reads are deposited at MG-RAST (Project ID: 152).

Subsequent feature selection was carried out using the Kruskal-Wallis test. For phylum, class, order and family taxonomic levels, correction for multiple testing was applied using Holm's method. ${ }^{26}$ For the species and genus level analysis, the less stringent method of Benjamini and Hochberg was used. ${ }^{27}$ This method controls the false discovery rate and so is a less stringent condition than the control of the family-wise error rate. When applying statistics, a cut-off of $20 \%$ occupancy of a taxa was used to remove rare or poorly measured species. This means that a variable was removed from testing if it contained 46 or more zeros. Testing of rates of depression across the IBS groups was carried out using the Kruskal-Wallis test and confirmed in a pairwise manner using the asymptotic Wilcoxon-Mann-Whitney rank sum test.

\section{Assigning species and Clostridium clusters}

To confidently assign amplicon sequences to species level and Clostridium clusters, we extracted 72928 V4 sequences from the 123332 full-length $16 \mathrm{~S}$ rRNA genes with complete species classifications (RDP release 10.23) using the same primer pair locations as were used for the amplification. In brief, an association table with species-specific cut-off BLAST scores was designed from an all-against-all BLAST search of the extracted $\mathrm{V} 4$ sequences. If the same-species score for a certain species was higher than the score of the first hit against a different species, then that species was considered assignable. The relaxed cut-off threshold was then set as the recorded score of the first hit against a different species, plus one. As this is less than the last true positive, it introduces a small degree of ambiguity. This was deemed necessary to maximise the number of species that could potentially be identified, especially in situations where a species had only one known V4 sequence. This methodology was applied at the level of OTU where the representative sequences were used to assign OTUs to species. In this way, a total of $32 \%$ of reads were classified to 168 species. To test each of these species, the associated OTUs were combined to generate a 168 * 57 dataset. A stricter cut-off score was also calculated as the score of the last same-species hit just prior to the first hit against a different species. The Clostridium clusters thresholds were defined in a similar way using combined all-against-all BLAST results from a list of species that were classified to Clostridium clusters according to the literature.

\section{RESULTS}

\section{Descriptive analysis}

Fifty-seven subjects were enrolled: 37 IBS (mean age $37 \pm 12$ years (mean $\pm S D$ ); 26 female subjects) and 20 non-IBS control subjects (mean age $39 \pm 9$ years; 13 female subjects). 
Based on Rome II criteria, ${ }^{8} 12$ of these subjects had alternatingtype IBS, 15 diarrhoea-predominant IBS and 10 constipationpredominant IBS. Data from questionnaires and physiological tests are summarised in online supplementary table 1. Time since first symptoms was $>5$ years in 35 of the 37 IBS patients and over a year in the remaining two. Using established $\mathrm{HAD}$ cut-off values for clinically significant $(\geq 11)$ anxiety or depression, 11 and 8 patients had clinically significant anxiety and depression, respectively (30\% and 22\%). Thirty-two (86\%) patients had SF-36 scores below 50 on the mental and/or physical component scores, indicating reduced quality of life. Based on normal values, ${ }^{21} 2211 / 36$ patients had rectal hypersensitivity, whereas colonic transit was rapid in 8 , slow in 3 and normal in 17 patients.

\section{Identification of IBS subtypes by cluster analysis}

Hierarchical clustering based on the Pearson correlation coefficient was applied to the OTU tables. This identified a readily discernible split for a subsection of the IBS subjects, with two clusters representing 22 of the 37 IBS microbiota datasets, separating from the control samples (figure 1A). These clusters were not associated with medication, body mass index (BMI), total read numbers or Rome II categories ( $p$ value $>0.05$ ). Weighted Unifrac principal coordinates analysis (PCoA) revealed that these two clusters were clearly separated from each other and the control samples and the remaining normal-like IBS samples (figure $1 \mathrm{~B}$ ).

Analysis of $\alpha$ diversity (ie, diversity within a single microbiota) showed the IBS subjects, as a whole, to have average microbiota diversity lower than healthy controls (figure 2A). However, when the analysis was applied in a group-wise manner (figure 2B), the normal-like IBS showed similar diversity levels as the controls and the two IBS clusters showed different levels of diversity. Cluster 1 showed a diminished diversity, while cluster 2 had increased diversity when compared with the control samples. This difference in diversity could also be seen in the number of species that could be confidently assigned, with a median of 44 in cluster 1 compared with 53 in cluster 2 . Differing depths of sequencing can be excluded as a reason for this observation since the average total read numbers were higher in cluster 1 than cluster 2, although this was not significant.

The separation of cluster 1 from cluster 2 was independent of the separation from the control samples along the first axis (figure 1B) but was significantly different from each other based on permutational multivariate analysis of variance (PMANOVA) with both weighted and unweighted Unifrac distances. We again applied the PMANOVA on the Unifrac distances and tested the two IBS clusters, the remaining IBS and the control samples against each other. The IBS clusters were significantly different from the remaining normal-like IBS samples, the control samples and each other (unweighted Unifrac: $p<0.005$, weighted Unifrac $\mathrm{p}<0.005)$. To further investigate clustering of subjects by their microbiota composition, the phylum and genus level datasets were visualised using pie charts (figure 3) and bar charts (online supplementary figure 1). The most striking feature was the change in the Firmicutes:Bacteroidetes (FB) ratio between the groups at the phylum level and the similarity of the normal-like IBS microbiota to the control samples, even at the genus level (figure 3, online supplementary figure 1). Hereinafter, the two IBS clusters will be collectively referred to as the high FB ratio IBS and the remaining IBS as normal-like IBS.

\section{Statistical analysis of the taxonomic level datasets}

Multigroup analysis of the defined groups identified a number of taxa that were present at significantly different relative proportions between the high FB ratio IBS clusters, the normallike IBS and the controls. These differences can be described as being between the high FB ratio IBS clusters and the controls, as the normal-like IBS showed similar proportions of these taxa to

A

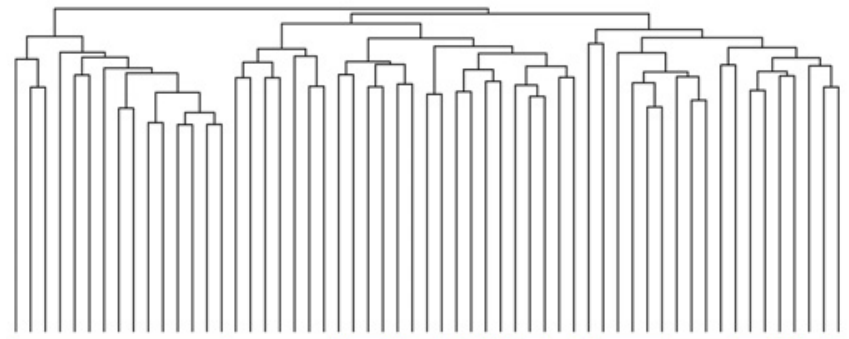
(1) Cluster 2 IBS
Cluster 1 IBS Control samples
Normal-like IBS

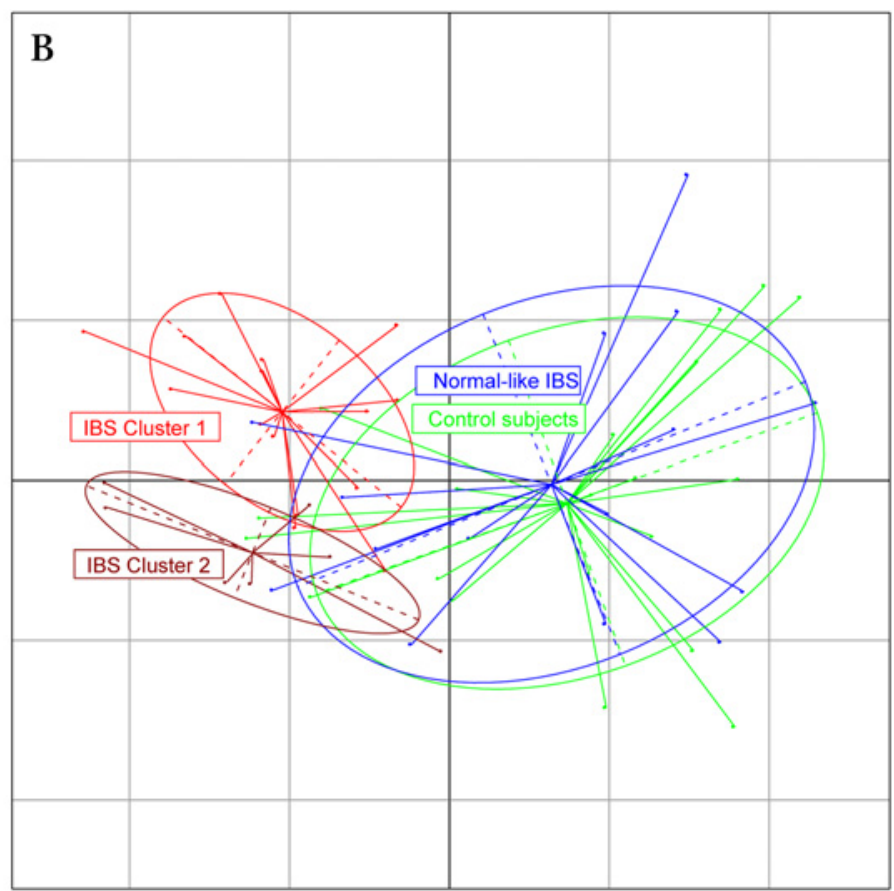

Figure 1 Clustering analysis of irritable bowel syndrome (IBS) and control samples. (A) Hierarchical trees based on scaled log transformed operational taxonomic unit data. (B) Principal coordinates analysis of weighted Unifrac distances showing the controls (green), high Firmicutes: Bacteroidetes (FB) ratio IBS (red) and normal FB ratio IBS (blue). 

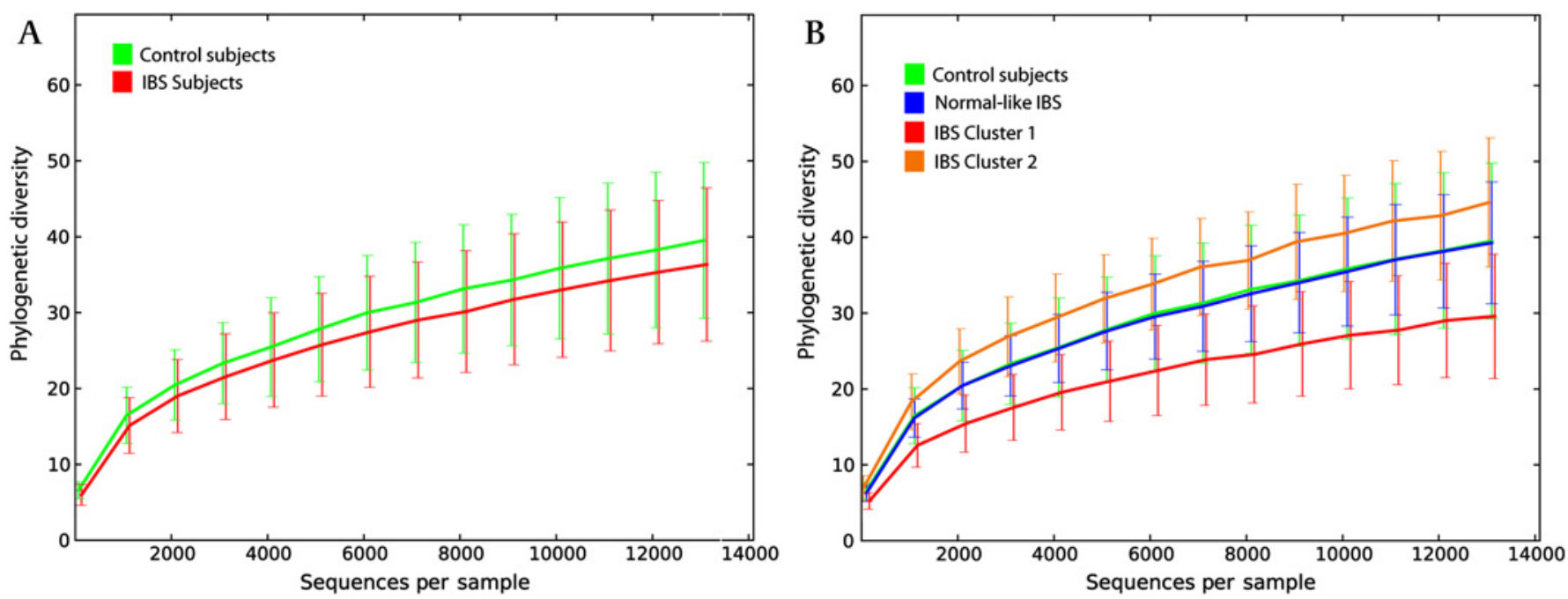

Figure 2 Faith's phylogenetic diversity curves. $\alpha$ Diversity curves showing total branch length with SDs for each group, for each number of sequences generated through rarefaction for (A) control and irritable bowel syndrome (IBS) samples and (B) control and IBS subgroups.

the controls with only slight differences. Taxa increased in the high FB ratio clusters were significantly enriched for Firmicutesassociated taxa (table 1 and online supplementary table_2). Bacteroidetes-associated taxa were decreased when compared with the control subjects' microbiota (table 2 and online supplementary table_2). The exception to this trend was Sporobacter which had highest abundance in IBS cluster 2 but was depleted in cluster 1. The most abundant Bacteroidetes-associated genera were depleted in both clusters (Bacteroides $(p<0.0001)$ and Alistipes $(\mathrm{p}=0.001))$.

Phylum level analysis showed an increase in Actinobacteria $(p<0.0001)$. Bifidobacteriaceae $(p<0.001)$ was associated with a median of $95 \%$ of the reads in the phylum Actinobacteria. This compared with Actinomycetaceae $(\mathrm{p}<0.001)$ and Coriobacteriaceae $(p<0.01)$ which were associated with less than $1 \%$ and $3 \%$ of reads, respectively. Further investigation of the Bifidobacterium result found that $95 \%$ of the associated reads mapped to one OTU that was present in all control and 36 of the 37 IBS samples. Performing a BLAST search using the 207 base pair representative sequence against the RDP database provided a $100 \%$ hit to Bifidobacterium adolescentis.

One genus that shows differential abundance in the data presented in figure 3 is Faecalibacterium. Faecalibacterium prausnitzii abundance was also investigated using targeted quantitative real-time PCR (online supplementary notes), in addition to the pyrosequencing analysis. In both datasets, there was a small increase in the abundance of this bacterium in the IBS samples but significance testing showed that this increase was well within the range of values expected, based on the high level of variance associated with the bacterium prevalence. Interestingly, the IBS sample variance was twice the variance in the control samples.

A total of 163 species were confidently identified as discussed in the Methods section. Of these, 11 species were associated with the separation of the high FB ratio IBS clusters from the healthy controls (table 3 ).
Figure 3 Visualisation of taxonomic levels. Pie charts showing proportion of reads in each phylum (top) and genus (bottom) for the controls, normal-like irritable bowel syndrome (IBS) and the IBS clusters.

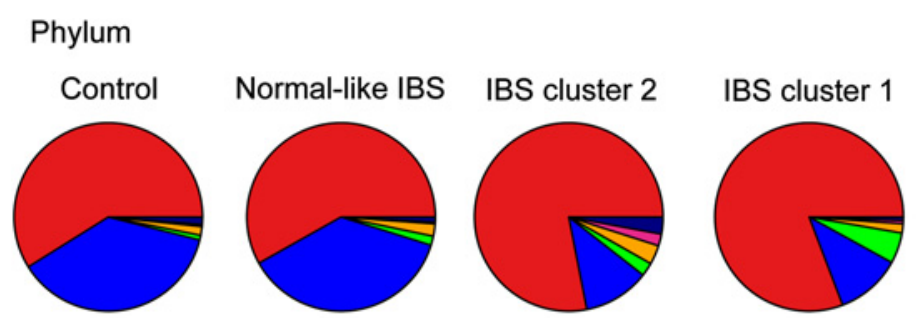

Genus
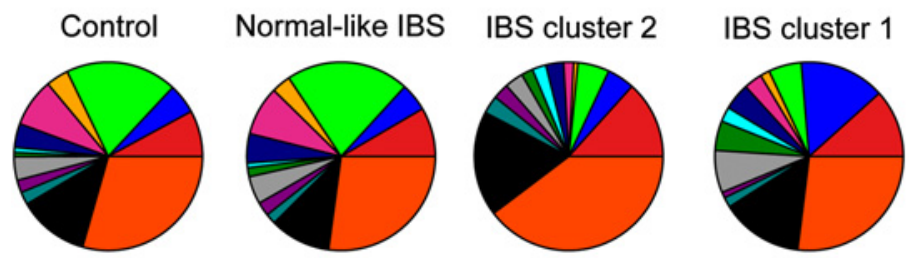



Faecalibacterium

- Blautia

$\square$ Bacteroides

$\square$ Parabacteroides

$\square$ Alistipes

- Ruminococcus

$\square$ Subdoligranulum

$\square$ Bifidobacterium

$\square$ Roseburia

- Oscillibacter

$\square$ Coprococcus

- Other

Unclassified 
Table 1 Increased abundance taxa associated with high Firmicutes:Bacteroidetes (FB) ratio irritable bowel syndrome (IBS)

\begin{tabular}{|c|c|c|c|c|c|}
\hline & Controls & Normal-like IBS & IBS cluster 1 & IBS cluster 2 & p Value \\
\hline \multicolumn{6}{|l|}{ Phylum } \\
\hline Firmicutes & $55.7(50-71.9)$ & $56.1(46.2-74)$ & $81.4(74.8-87.7)$ & $80.4(71-83.6)$ & $<0.0001$ \\
\hline Actinobacteria & $0.75(0.27-1.14)$ & $0.92(0.15-1.88)$ & $5.38(3.69-7.18)$ & $2.29(1.64-2.55)$ & $<0.0001$ \\
\hline Clostridia & $54.3(46.6-67.3)$ & $54(44.6-68.9)$ & $74.2(71.5-81.7)$ & $74.5(65.3-79.2)$ & $<0.001$ \\
\hline Actinobacteria & $0.75(0.27-1.14)$ & $0.92(0.15-1.88)$ & $5.38(3.69-7.18)$ & $2.29(1.64-2.55)$ & $<0.0001$ \\
\hline Erysipelotrichi & $0.8(0.5-1.62)$ & $1.26(0.76-1.37)$ & $2.17(0.97-5.89)$ & $1.9(1.53-4.51)$ & $<0.01$ \\
\hline Clostridiales & $54.2(46.4-67.1)$ & $53.7(44.5-68.6)$ & $74(71.4-81.7)$ & $74.2(65.1-78.8)$ & $<0.001$ \\
\hline Bifidobacteriales & $0.71(0.27-1.03)$ & $0.9(0.13-1.82)$ & $5.08(2.98-7.14)$ & $1.55(1.35-2.35)$ & $<0.001$ \\
\hline Enterobacteriales & $0.0047(0-0.0081)$ & $0.0067(0.0037-0.011)$ & $0.036(0.009-0.087)$ & $0.19(0.13-0.5)$ & $<0.001$ \\
\hline Actinomycetales & $0.008(0.004-0.016)$ & $0.008(0.005-0.016)$ & $0.03(0.015-0.04)$ & $0.022(0.02-0.047)$ & $<0.001$ \\
\hline Coriobacteriales & $0.014(0.007-0.052)$ & $0.013(0.007-0.025)$ & $0.0435(0.01-0.13)$ & $0.214(0.161-0.308)$ & $<0.01$ \\
\hline Bacillales & $0(0-0)$ & $0(0-0)$ & $0.004(0-0.0067)$ & $0(0-0.00195)$ & $<0.01$ \\
\hline Actinomycetaceae & $0.0076(0.0036-0.0139)$ & $0.0077(0.0034-0.0133)$ & $0.03(0.0147-0.0381)$ & $0.0217(0.018-0.0405)$ & $<0.001$ \\
\hline Staphylococcaceae & $0(0-0)$ & $0(0-0)$ & $0.0034(0-0.0055)$ & $0(0-0)$ & $<0.01$ \\
\hline Coriobacteriaceae & $0.014(0.007-0.052)$ & $0.013(0.007-0.025)$ & $0.0435(0.01-0.13)$ & $0.214(0.161-0.308)$ & $<0.01$ \\
\hline Incertae sedis XII & $0(0-0.0063)$ & $0.0101(0-0.03)$ & $0(0-0)$ & $0.0087(0-0.0458)$ & $<0.01$ \\
\hline \multicolumn{6}{|l|}{ Genus } \\
\hline Papillibacter & $0.14(0.0875-0.28)$ & $0.196(0.14-0.282)$ & $0.542(0.44-0.701)$ & $0.186(0.156-0.255)$ & $<0.0001$ \\
\hline Dialister & $0.029(0-0.128)$ & $0.061(0.0056-0.747)$ & $1.47(0.782-4.07)$ & $0.441(0.029-0.611)$ & $<0.0001$ \\
\hline Bifidobacterium & $0.71(0.27-1.02)$ & $0.894(0.125-1.81)$ & $5.07(2.97-7.12)$ & $1.54(1.34-2.32)$ & $<0.001$ \\
\hline Dorea & $0.566(0.269-0.845)$ & $0.395(0.315-0.56)$ & $1.71(0.925-2.91)$ & $0.593(0.433-1.03)$ & $<0.001$ \\
\hline Blautia & $3.83(2.88-8.18)$ & $5.12(3.47-5.45)$ & $14.1(7.18-20.3)$ & $4.69(3.86-5.69)$ & $<0.001$ \\
\hline Sporobacter* & $0.837(0.246-1.65)$ & $0.805(0.402-1.02)$ & $0.0615(0.015-0.4)$ & $2.22(1.59-2.5)$ & $<0.001$ \\
\hline Actinomyces & $0.006(0.001-0.014)$ & $0.0077(0.0034-0.012)$ & $0.03(0.015-0.036)$ & $0.019(0.018-0.034)$ & $<0.001$ \\
\hline
\end{tabular}

Significant results from the four group Kruskal-Wallis analysis.

This table represents the taxa that have higher abundance in the high FB ratio IBS clusters when compared with the control samples.

Values are shown as the median and associated IQR for the percentage of reads for the taxa in each group.

${ }^{*}$ Sporobacter has both highest and lowest values in the IBS clusters but is presented in this table as having its highest value in IBS cluster 2.

Statistical analysis of the combined abundances of species that belong to the Clostridium clusters identified Clostridium cluster XIVa as being significantly differently abundant across the groups, specifically characterised with an increased abundance in IBS cluster $1(\mathrm{p}<0.0005)$.

\section{OTU signatures for IBS subtypes}

Statistical analysis identified 111 OTUs that were significantly differentially abundant across the four groups (online supplementary table_3). Phylogenetic assignments assigned the vast majority of the OTUs that had higher abundances in the high

Table 2 Increased abundance taxa associated with control samples

\begin{tabular}{|c|c|c|c|c|c|}
\hline & Controls & Normal-like IBS & IBS cluster 1 & IBS cluster 2 & p Value \\
\hline \multicolumn{6}{|l|}{ Phylum } \\
\hline Bacteroidetes & $40.6(25.4-45.8)$ & $40.1(22-50)$ & $13.8(3.98-16.3)$ & $10.2(5.45-17.4)$ & $<0.0001$ \\
\hline \multicolumn{6}{|l|}{ Class } \\
\hline Bacteroidia & $38.9(25.3-44.5)$ & $40.1(18.3-49.9)$ & $13.7(3.96-15.8)$ & $10(5.02-14.7)$ & $<0.0001$ \\
\hline \multicolumn{6}{|l|}{ Order } \\
\hline Bacteroidales & $38.9(25.3-44.5)$ & $40.1(18.3-49.9)$ & $13.7(3.96-15.8)$ & $10(5.02-14.7)$ & $<0.0001$ \\
\hline \multicolumn{6}{|l|}{ Family } \\
\hline Bacteroidaceae & $16.2(10.2-23.9)$ & $17.8(8.91-32.5)$ & $5.55(2.54-8.41)$ & $3.83(1.73-9.26)$ & $<0.0001$ \\
\hline Porphyromonadaceae & $6.1(3.44-9.86)$ & $3.42(2.38-6.44)$ & $1.53(0.37-2.37)$ & $1.59(0.575-2.06)$ & $<0.001$ \\
\hline Rikenellaceae & $7.84(5.87-10.8)$ & $7.03(4.29-10.8)$ & $1.84(0.49-4.66)$ & $1.6(0.77-1.93)$ & $<0.001$ \\
\hline \multicolumn{6}{|l|}{ Genus } \\
\hline Odoribacter & $0.3(0.123-0.577)$ & $0.147(0.068-0.278)$ & $0.013(0.002-0.042)$ & $0.104(0.045-0.227)$ & $<0.0001$ \\
\hline Bacteroides & $16.2(10.2-23.9)$ & $17.8(8.91-32.5)$ & $5.55(2.54-8.41)$ & $3.83(1.73-9.26)$ & $<0.0001$ \\
\hline Alistipes & $7.78(5.7-10.3)$ & $6.99(4.23-10.8)$ & $1.78(0.491-4.5)$ & $1.4(0.707-1.91)$ & $<0.001$ \\
\hline
\end{tabular}

Significant results from the four group Kruskal-Wallis analysis.

This table represents the taxa that have higher abundance in the control samples when compared with the high Firmicutes:Bacteroidetes ratio IBS clusters.

Values are shown as the median and associated IQR for the percentage of reads for the taxa in each group.

IBS, irritable bowel syndrome. 
Table 3 Species associated with high Firmicutes:Bacteroidetes (FB) ratio irritable bowel syndrome (IBS) versus control split

\begin{tabular}{|c|c|c|c|c|c|}
\hline Species & Controls & Normal-like IBS & IBS cluster 1 & IBS cluster 2 & p Value \\
\hline \multicolumn{6}{|c|}{ Most abundant in high FB ratio IBS-associated } \\
\hline Eubacterium hallii & $0.74(0.42-1.2)$ & $0.91(0.78-1.52)$ & $4.59(2.24-7.08)$ & $0.97(0.57-1.66)$ & $<0.0001$ \\
\hline Clostridium innocuum & $0.0048(0-0.0087)$ & $0.0078(0.0034-0.0144)$ & $0.0428(0.0242-0.0936)$ & $0(0-0.004)$ & $<0.0001$ \\
\hline Eubacterium desmolans & $0.095(0.075-0.172)$ & $0.15(0.053-0.258)$ & $0.398(0.222-0.454)$ & $0.282(0.133-0.767)$ & $<0.01$ \\
\hline Eubacterium eligens & $0.483(0.174-0.71)$ & $0.298(0.015-0.834)$ & $0.0035(0-0.0456)$ & $1.75(0.0397-4.12)$ & $<0.01$ \\
\hline Eubacterium siraeum* ${ }^{*}$ & $0.339(0.028-1.16)$ & $0.285(0.017-1.26)$ & $0.0043(0-0.0182)$ & $0.457(0.146-1.35)$ & $<0.01$ \\
\hline Alistipes putredinis & $3.44(2.03-6.31)$ & $2.79(1.67-4.45)$ & $0.59(0.007-1.55)$ & $0.503(0.095-1.15)$ & $<0.001$ \\
\hline Barnesiella intestinihominis & $0.87(0.24-1.92)$ & $0.22(0.007-0.489)$ & $0.0027(0-0.243)$ & $0.26(0.1-0.67)$ & $<0.001$ \\
\hline Bacteroides caccae & $0.33(0.1-1.05)$ & $0.28(0.006-0.596)$ & $0(0-0.052)$ & $0.1(0.018-0.175)$ & $<0.01$ \\
\hline Bacteroides stercoris & $0.025(0.0014-0.7)$ & $0(0-0.0037)$ & $0(0-0)$ & $0(0-0.0153)$ & $<0.01$ \\
\hline
\end{tabular}

Species showing significant differences in abundance across the four groups as defined from the Kruskal-Wallis analysis.

Values are shown as the median and associated IOR for the percentage of reads for the taxa in each group.

* Sporobacter termitidis and Eubacterium siraeum have both the highest and lowest values in the IBS clusters but are presented in this table as having their highest values in IBS cluster 2.

FB ratio clusters to Firmicutes-associated taxa. Bacteroidetesassociated taxa were associated with OTUs that were more abundant in the control samples.

\section{Correlation of differential microbiota composition with clinical data in IBS}

Associations between the clinical data and composition of the microbiota were tested with the Kendall rank correlation coefficient within all IBS subjects and separately for the high FB IBS subjects, and for each of the subgroups. The significant correlations are reported in table 4 . In total, eight clinical variables showed associations across 21 taxa over the four groups of subjects tested. Two phylum level associations that were returned as significant involved multiple clinical characteristics in the analyses. An increased abundance of Cyanobacteria was associated with satiety, bloating and an increased total gastrointestinal symptom rating scale-IBS (GSCR-IBS) score. Proteobacteria abundance was associated with an increased mental component and an increased pain threshold.

Another 17 taxa were associated with prolonged colonic transit times. These taxa can be summarised to two species and three families that were associated with a prolongation of colonic transit time and constipation. The order Actinomycetales and the family Actinomycetaceae were inversely associated with clinically significant depression indicating that people with IBS without clinically significant depression carry a greater load of this bacterium. Interestingly, the normal subjects carry significantly less of this bacterium than the non-depressed IBS ( $p$ value: 0.0017 )

\section{Clinical variables and IBS subtypes}

The rate of clinically significant depression in the high FB ratio IBS clusters was $2 / 22$ (9\%). This rate of clinically significant depression is comparable with that in the general Swedish population. ${ }^{28}$ This compares with the normal-like IBS group at $6 / 15$ subjects or $40 \%$. When tested using the HAD depression scores, it was found that the difference was significant based on the pairwise test between high FB ratio IBS clusters and normallike IBS $(p=0.02)$. The HAD depression score was the most differentiating clinical variable between the high FB ratio IBS and the normal-like IBS, evidenced by relationships between the microbiota and clinical variables, when visualised by correspondence analysis (figure 4). Correspondence analysis also showed a high correlation between clinically significant depres- sion and anxiety $(\mathrm{r}=0.65, \mathrm{p}$ value $<0.00005)$. Other variables were not as discriminating between the two groups, but there are trends towards higher mental and physical component scores (SF-36) and rectal discomfort and pain thresholds in the high FB ratio IBS versus the normal-like IBS group ( $p>0.05)$.

\section{DISCUSSION}

We examined relationships between microbiota composition and clinical and physiological parameters in a cohort of well-characterised IBS patients, potentially facilitating mechanistic insights. Not surprisingly, given the heterogeneity that is intrinsic to IBS, clustering analysis of the microbiota data revealed different populations of IBS subjects. These groups were significantly different from each other. The first of these groups $(n=15)$ clustered with the control samples and had no significantly associated microbiota composition features when compared with non-IBS controls. The second group consisted of two clusters (figure 1) that separated from the control samples. These IBS samples $(n=22)$ were defined by large microbiota-wide changes. A number of associations were detected between microbiota composition and clinical or physiological phenotypes.

Previous studies have shown that the majority of the IBS samples formed a distinct cluster. ${ }^{10} 29$ However, to the best of our knowledge, this is the first study to robustly define IBS patients with no detectable changes in their faecal microbiota.

\section{Correlation of differential microbiota composition with clinical data in IBS}

Of the various parameters examined, the presence or absence of clinically significant depression was the single clinical feature that segregated in parallel with microbiota composition findings. Thus, while co-morbid depression was common among the normal-like IBS group, the prevalence of clinically significant depression among the high FB clusters was akin to the rate in the general population. While this observation deserves further study and requires confirmation, it does tempt one to speculate that microbiota analysis may permit differentiation of IBS patients into two groups: those in whom psychological comorbidity is highly prevalent and those in whom a more 'organic' pathophysiology, such as microbe-host immune interaction, may be more operative.

Statistical analysis showed that certain bacterial phylotypes were associated with clinical markers of IBS. Analysis of the IBS microbiota and separate analyses of the two subgroups showed 
Table 4 Associations between clinical characteristics of irritable bowel syndrome (IBS) and taxa

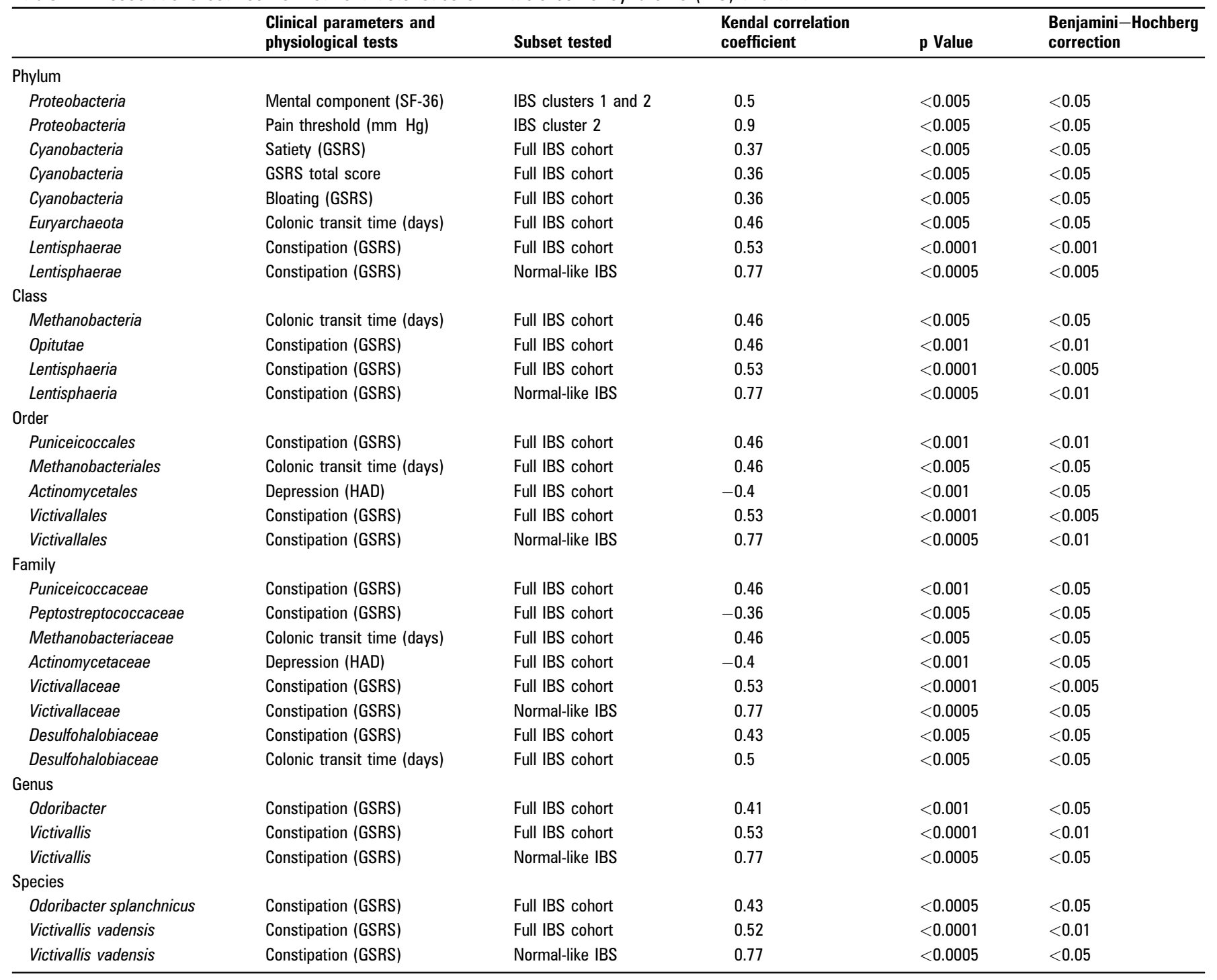

Significant results from analysis of subsets of IBS patients.

Values are correlations between the taxa and clinical parameters and physiological tests.

Pain thresholds are based on the rectal sensitivity test.

GSRS, Gastrointestinal Symptom Rating Scale; HAD, Hospital Anxiety and Depression Scale; SF-36, short form with 36 quality of life questions.

microbial associations with colonic transit time, satiety, bloating, rectal pain threshold and depression.

\section{Comparison with previous studies}

Other groups have observed a significantly lower proportion of Bacteroides spp. in IBS patients than in healthy controls, but at the Bacteroidetes phylum level, no difference was detected between the groups. ${ }^{10} 30$ In the present study, we observed a reduction of Bacteroidetes that was confined to a high FB ratio subset of IBS patients. There was a general decrease in the two most common genera (Bacteroides and Alistipes). Similarly, at the OTU level, the Bacteroidetes-related genera involved were identified as Bacteroides, Alistipes and Parabacteroides.

Increased Firmicutes abundance has previously been recorded in IBS subjects. ${ }^{10-12} 15$ In our current study, this was combined with a decrease in the abundance of Bacteroidetes. This increase in the ratio of Firmicutes to Bacteroidetes was associated with over half of the IBS samples studied. The remaining normal FB ratio IBS samples had no detectable changes in the faecal microbiota.
In common with previous studies using molecular methods, we sampled the IBS microbiota at a single time-point. The intestinal microbiota is stable in healthy adults, including older adults in whom the microbiota is considered to be in flux, ${ }^{23}$ but it will be interesting in future studies to monitor the microbiota of IBS patients in sequential samples, including those which cover periods of remission, relapse and changes in bowel function.

\section{Inflammation-linked microbiota}

Elevated levels of serum antibodies specific for bacterial flagellins have been detected in postinfectious IBS patients. ${ }^{31}$ The possibility that flagellin-producing species might be relevant to the pathophysiology of IBS is supported by the documentation of a low level of mucosal inflammation within the GI tract, as well as a systemic pro-inflammatory cytokine phenotype, in all subtypes of IBS. ${ }^{32}$ Furthermore, though postinfectious IBS only explains a minority of cases of IBS, the documentation of the occurrence of IBS following bacteriologically-confirmed gastroenteritis in several studies ${ }^{2-4}$ represents a clear link 
Figure 4 Clinical information. Correspondence analysis showing high Firmicutes:Bacteroidetes (FB) ratio irritable bowel syndrome (IBS) (red) and normal-like IBS (blue) patients in the top panel and the clinical variables in the lower panel. GSRS, Gastrointestinal Symptom Rating Scale; HAD, Hospital Anxiety and Depression Scale; SF-36, short-form 36 .

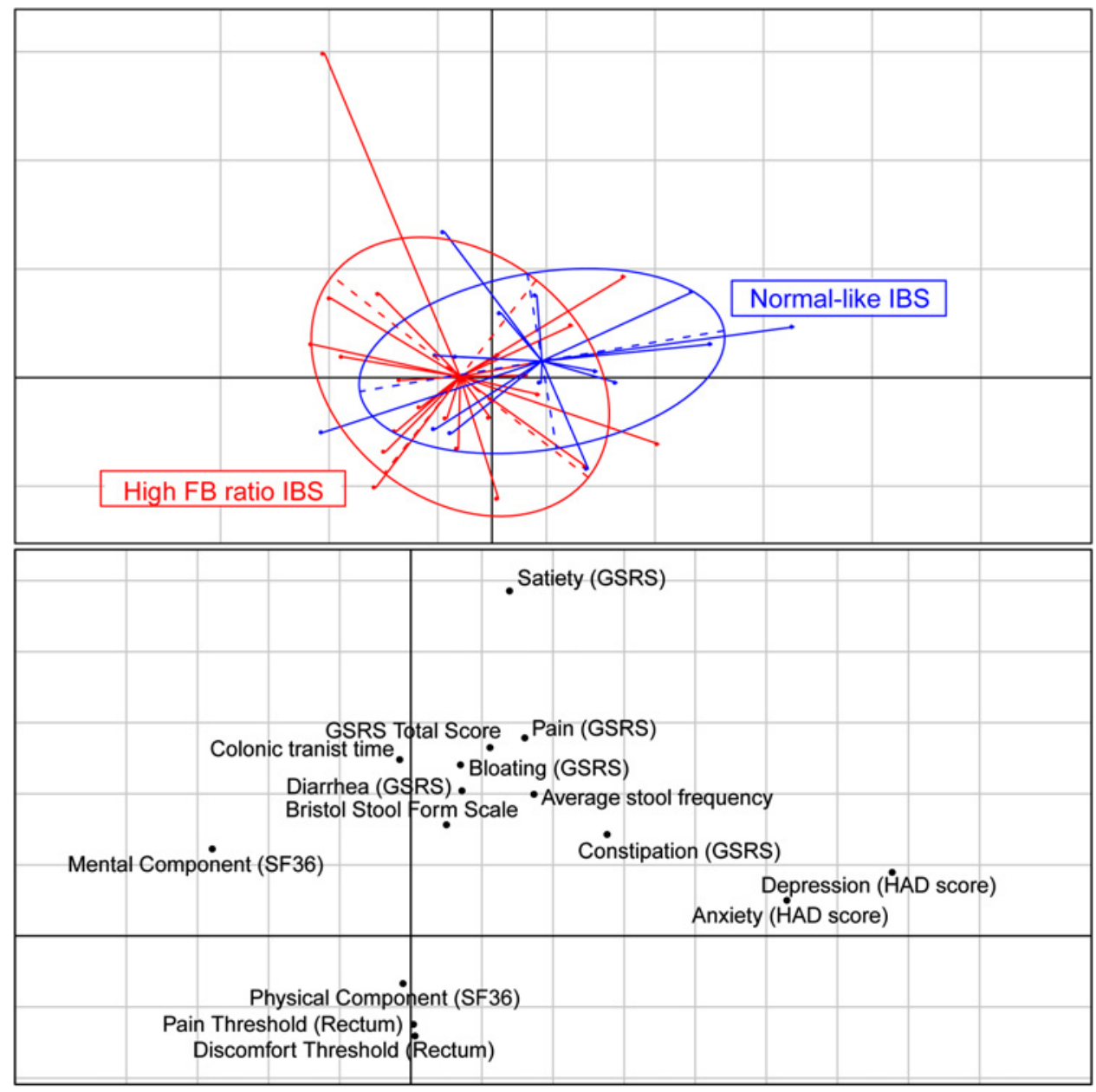

between exposure to a bacterium, a sustained inflammatory response and IBS.

Of the 73 OTUs of the phylum Firmicutes that were differentially expressed across the groups, 70 were Clostridia/Clostridiales. The genus Lachnospiraceae incertae sedis accounted for 18 of these and has been shown to be enriched in diarrhoeapredominant IBS patients. ${ }^{33}$ Species belonging to $L$ incertae sedis are known to express inflammatory flagellin proteins. ${ }^{34}$ These inflammatory flagellin-producing species were also classified as Clostridium cluster XIVa.

Clostridium cluster XIVa has also previously been shown to be associated with IBS. ${ }^{10}$ Our study noted an increased abundance of Clostridium cluster XIVa as being associated with the high FB ratio IBS.

\section{Short-chain fatty acid producers and IBS}

A number of studies have shown an increase in the amount of short-chain fatty acids (SCFAs) in IBS. ${ }^{15} 35$ Butyrate and acetate have many beneficial effects in relation to oxidative stress and inflammation, ${ }^{36}$ energy for the colonic epithelium, ${ }^{37}$ modulation of cell proliferation ${ }^{38}$ and protection against GI infections. ${ }^{39} 40$ However, butyrate promoted visceral hypersensitivity in a rat model $^{41}$ and the butyrate-producing associated Clostridium cluster XIVa group ${ }^{42}$ and some specific butyrate-producing species such as Eubacterium hallii and Eubacterium desmolans ${ }^{43}$ were associated with IBS in this study. Taken together, these findings could explain the sensory dysfunction and poor tolerance of gas retention associated with IBS. ${ }^{44}$

Perhaps of greater significance is that the production of SCFAs has the ability to lower the $\mathrm{pH}$ in the colon. ${ }^{45}$ Duncan et al showed that tolerance of a reduced $\mathrm{pH}$ among the Firmicutes spp. varied for different species but, overall, the Clostridium cluster XIVa group showed the smallest reduction in growth rate, while the butyrate-producing associated Clostridium cluster IV growth rates were dramatically reduced. ${ }^{45}$ We detected no significant change in the overall abundance of Clostridium cluster IV.

$B$ adolescentis are prominent in the adult gut microbiota. $B$ adolescentis produces acetic acid and lactate, indicating it as a potential source of the increased SCFAs in IBS. ${ }^{46}$ Although we did not control for probiotics and probiotic use may have occurred in this population, we believe it unlikely that this increase in $B$ adolescentis originated from an extraneous origin. Duncan et $a l^{45}$ and Collado and Sanz ${ }^{47}$ tested the tolerance of Bifidobacterium spp. to acidic $\mathrm{pH}$ conditions and showed that $B$ adolescentis showed no deterioration in their growth rate but other species, including more abundant species, showed a reduced abundance when exposed to acidic conditions. These observations may explain why we recorded a significant increase in $B$ adolescentis but not other species of Bifidobacterium in our analysis and the apparent disagreement with previous studies that demonstrated a reduction in Bifidobacterium, particularly $B$ catenulatum, in IBS. ${ }^{13} 1448$ It is unclear why our procedure apparently favoured detection of $B$ adolescentis above other species, but differences in primer binding, amplification or DNA extraction efficiency for each species were likely contributors.

The reduction of Bacteroides proportion has been noted in this study and, interestingly, has also been associated with IBS. ${ }^{49}$ Bacteroidetes spp. experience progressive inhibition at reduced $\mathrm{pH}$ values. ${ }^{45}$ At least one Bacteroides spp. is known to have beneficial effects on mucosal tolerance. ${ }^{50}$ 


\section{Antibiotic or probiotic consumption in IBS}

We controlled for antibiotic use within 1 month of faecal sampling. While most constituents of the gut microbiota return to preantibiotic levels within 4 weeks, we concede that recent data shows that some species may fail to recover to preantibiotic levels for much longer periods after the end of antibiotic therapy. ${ }^{51}$ Given these findings, and the knowledge that Bifidobacterium spp. and some Bacteroides spp. that have been shown to be associated with IBS may be especially sensitive to longer-term effects of antibiotic use, we would advocate more prolonged antibiotic-free periods prior to faecal sampling in future studies. However, given the ubiquity of antibiotic use in the community and the advocacy, by some, of antibiotics for the treatment of IBS, this may be challenging in practice. We did not control for probiotic consumption, which might theoretically cause confounding effects upon microbiota composition. However, recent studies in our laboratory (Riboulet-Bisson, O'Toole et al, submitted) show very modest effects on the intestinal microbiota of mice and pigs administered large doses of bacteriocinproducing probiotic lactobacilli, and the effects in humans are also modest. ${ }^{5253}$

\section{CONCLUSION}

Recently, the microbiota in IBS has been linked to immunological alterations and low grade inflammation in IBS. This study used a comparatively large number of well-characterised IBS subjects and deep level pyrosequencing to identify candidate microbiota elements that contribute to the pathology of IBS. This is the first study to define patient subgroups whose microbiota had markedly different characteristics and to associate microbial signatures to clinical variables within these subgroups. We have extended previous studies and provided new bacterial gut signatures and associations; some will require further investigation to fully define their role in IBS.

Funding This study was supported by a Science Foundation Ireland CSET award to the Alimentary Pharmabiotic Centre, by the (Government of Ireland) Department of Agriculture Fisheries and Food/Health Research Board FHRI award to the ELDERMET project, by the Swedish Medical Research Council (grants 13409, 21691 and 21692), by the Marianne and Marcus Wallenberg Foundation and by the Faculty of Medicine, University of Gothenburg. MJC is supported by a fellowship from the Health Research Board of Ireland.

\section{Competing interests None}

\section{Patient consent Obtained.}

Ethics approval This study was approved by the Regional Ethical Review Board at the University of Gothenburg.

Contributors IBJ: analysis and interpretation of data, drafting of the manuscript and statistical analysis. PWO'T: study concept and design, critical revision of the manuscript for important intellectual content and obtained funding. LÖ: acquisition of data. MJC: analysis and interpretation of data. JD: technical or material support. EMMQ: study concept and design and critical revision of the manuscript for important intellectual content. MS: study concept and design, critical revision of the manuscript for important intellectual content and obtained funding.

Provenance and peer review Not commissioned; externally peer reviewed.

Data sharing statement All sequence reads are deposited at MG-RAST laccession numbers pending).

\section{REFERENCES}

1. Paré $\mathbf{P}$, Gray J, Lam S, et al. Health-related quality of life, work productivity, and health care resource utilization of subjects with irritable bowel syndrome: baseline results from LOGIC (Longitudinal Outcomes Study of Gastrointestinal Symptoms in Canada), a naturalistic study. Clin Ther 2006;28:1726-35; discussion 1710-11.

2. Sethi S, Garey KW, Arora V, et al. Increased rate of irritable bowel syndrome and functional gastrointestinal disorders after Clostridium difficile infection. J Hosp Infect 2011;77:172-3
3. Ji S, Park H, Lee D, et al. Post-infectious irritable bowel syndrome in patients with Shigella infection. J Gastroenterol Hepatol 2005;20:381-6.

4. Hanevik K, Dizdar V, Langeland N, et al. Development of functional gastrointestinal disorders after Giardia lamblia infection. BMC Gastroenterol 2009;9:27.

5. Posserud I, Stotzer PO, Björnsson ES, et al. Small intestinal bacterial overgrowth in patients with irritable bowel syndrome. Gut 2007;56:802-8.

6. Pimentel M, Lembo A, Chey WD, et al. Rifaximin therapy for patients with irritable bowel syndrome without constipation. N Engl J Med 2011;364:22-32.

7. Tack J. Antibiotic therapy for the irritable bowel syndrome. N Engl J Med 2011;364:81-2.

8. Longstreth GF, Thompson WG, Chey WD, et al. Functional bowel disorders. Gastroenterology 2006;130:1480-91.

9. Salonen A, de Vos WM, Palva A. Gastrointestinal microbiota in irritable bowel syndrome: present state and perspectives. Microbiology 2010;156:3205-15.

10. Rajilić-Stojanović M. Diversity of the Human Gastrointestinal Microbiota-Novel Perspectives from High Throughput Analyses. [PhD thesis]. Wageningen, The Netherlands: Wageningen University, 2007.

11. Krogius-Kurikka L, Lyra A, Malinen E, et al. Microbial community analysis reveals high level phylogenetic alterations in the overall gastrointestinal microbiota of diarrhoea-predominant irritable bowel syndrome sufferers. BMC Gastroenterol 2009; $9: 95$.

12. Kassinen A, Krogius-Kurikka L, Mäkivuokko H, et al. The fecal microbiota of irritable bowel syndrome patients differs significantly from that of healthy subjects. Gastroenterology 2007;133:24-33.

13. Malinen E, Rinttilä T, Kajander K, et al. Analysis of the fecal microbiota of irritable bowel syndrome patients and healthy controls with real-time PCR. Am J Gastroenterol 2005;100:373-82.

14. Lyra A, Rinttilä T, Nikkilä J, et al. Diarrhoea-predominant irritable bowel syndrome distinguishable by $16 \mathrm{~S}$ rRNA gene phylotype quantification. World J Gastroenterol 2009;15:5936-45.

15. Tana C, Umesaki Y, Imaoka A, et al. Altered profiles of intestinal microbiota and organic acids may be the origin of symptoms in irritable bowel syndrome. Neurogastroenterol Motil 2010;22:512-19, e114-15.

16. Thompson WG, Longstreth GF, Drossman DA, et al. Functional bowel disorders and functional abdominal pain. Gut 1999;45(Suppl 2):॥43-7.

17. Zigmond AS, Snaith RP. The hospital anxiety and depression scale. Acta Psychiatr Scand 1983:67:361-70.

18. Wiklund IK, Fullerton S, Hawkey CJ, et al. An irritable bowel syndrome-specific symptom questionnaire: development and validation. Scand J Gastroenterol 2003;38:947-54

19. O'Donnell LJ, Virjee J, Heaton KW. Detection of pseudodiarrhoea by simple clinica assessment of intestinal transit rate. BMJ 1990;300:439-40.

20. Ware JE, Sherbourne CD. The MOS 36-item short-form health survey (SF-36). I. conceptual framework and item selection. Med Care 1992;30:473-83.

21. Posserud I, Syrous A, Lindström L, et al. Altered rectal perception in irritable bowel syndrome is associated with symptom severity. Gastroenterology 2007:133:1113-23.

22. Sadik R, Abrahamsson H, Stotzer PO. Gender differences in gut transit shown with a newly developed radiological procedure. Scand J Gastroenterol 2003;38:36-42.

23. Claesson MJ, Cusack S, O'Sullivan O, et al. Composition, variability, and tempora stability of the intestinal microbiota of the elderly. Proc Natl Acad Sci USA 2011;108 (Suppl 1):4586-91.

24. Cole JR, Wang 0 , Cardenas $\mathrm{E}$, et al. The Ribosomal Database Project: improved alignments and new tools for rRNA analysis. Nucleic Acids Res 2009;37:D141-5.

25. Lozupone C, Knight R. UniFrac: a new phylogenetic method for comparing microbia communities. Appl Environ Microbiol 2005;71:8228-35.

26. Holm SA. Simple sequentially rejective multiple test procedure. SCAND J STAT 1979;6:65-70.

27. Benjamini $\mathbf{Y}$, Hochberg $Y$. Controlling the false discovery rate: a practical and powerful approach to multiple testing. J ROY STAT SOC B MET 1995; 57:289-300.

28. Nationella Riktlinjer för Depressionssjukdom Och Ångestsyndrom—Beslutsstöd för Prioriteringar. Stockholm: The Swedish National Board of Health and Welfare, 2009

29. Codling C, O'Mahony L, Shanahan F, et al. A molecular analysis of fecal and mucosal bacterial communities in irritable bowel syndrome. Dig Dis Sci 2010:55:392-7.

30. Noor SO, Ridgway K, Scovell L, et al. Ulcerative colitis and irritable bowel patients exhibit distinct abnormalities of the gut microbiota. BMC Gastroenterol 2010:10:134

31. Schoepfer AM, Schaffer T, Seibold-Schmid B, et al. Antibodies to flagellin indicate reactivity to bacterial antigens in IBS patients. Neurogastroenterol Motil 2008:20:1110-18.

32. Ohman L, Simrén M. Pathogenesis of IBS: role of inflammation, immunity and neuroimmune interactions. Nat Rev Gastroenterol Hepatol 2010; 7:163-73.

33. Frank DN, St Amand AL, Feldman RA, et al. Molecular-phylogenetic characterization of microbial community imbalances in human inflammatory bowel diseases. Proc Natl Acad Sci USA 2007;104:13780-5

34. Duck LW, Walter MR, Novak J, et al. Isolation of flagellated bacteria implicated in Crohn's disease. Inflamm Bowel Dis 2007:13:1191-201.

35. Treem WR, Ahsan N, Kastoff G, et al. Fecal short-chain fatty acids in patients with diarrhea-predominant irritable bowel syndrome: in vitro studies of carbohydrate fermentation. J Pediatr Gastroenterol Nutr 1996;23:280-6. 
36. Vanhoutvin SALW, Troost FJ, Kilkens TOC, et al. The effects of butyrate enemas on visceral perception in healthy volunteers. Neurogastroenterol Motil 2009:21:952-e76.

37. Bugaut $\mathbf{M}$, Bentéjac M. Biological effects of short-chain fatty acids in nonruminant mammals. Annu Rev Nutr 1993;13:217-41.

38. Siavoshian S, Blottiere HM, Cherbut $\mathrm{C}$, et al. Butyrate stimulates cyclin D and $\mathrm{p} 21$ and inhibits cyclin-dependent kinase 2 expression in HT-29 colonic epithelial cells. Biochem Biophys Res Commun 1997;232:169-72.

39. Tedelind S, Westberg F, Kierrulf M, et al. Anti-inflammatory properties of the shortchain fatty acids acetate and propionate: a study with relevance to inflammatory bowel disease. World J Gastroenterol 2007;13:2826-32.

40. Fukuda S, Toh $\mathrm{H}$, Hase $\mathrm{K}$, et al. Bifidobacteria can protect from enteropathogenic infection through production of acetate. Nature 2011;469:543-7.

41. Bourdu S, Dapoigny M, Chapuy $\mathrm{E}$, et al. Rectal instillation of butyrate provides a novel clinically relevant model of noninflammatory colonic hypersensitivity in rats. Gastroenterology 2005;128:1996-2008.

42. Louis $\mathbf{P}$, Young $P$, Holtrop $G$, et al. Diversity of human colonic butyrate-producing bacteria revealed by analysis of the butyryl-CoA:acetate CoA-transferase gene. Environ Microbiol 2010;12:304-14.

43. Hold GL, Schwiertz A, Aminov Rl, et al. Oligonucleotide probes that detect quantitatively significant groups of butyrate-producing bacteria in human feces. App/ Environ Microbiol 2003;69:4320-4.

44. Serra J, Villoria A, Azpiroz F, et al. Impaired intestinal gas propulsion in manometrically proven dysmotility and in irritable bowel syndrome. Neurogastroenterol Motil 2010;22:401-6, e91-2.
45. Duncan SH, Louis $\mathrm{P}$, Thomson JM, et al. The role of $\mathrm{pH}$ in determining the species composition of the human colonic microbiota. Environ Microbiol 2009;11:2112-22.

46. Duncan SH, Louis P, Flint HJ. Lactate-utilizing bacteria, isolated from human feces, that produce butyrate as a major fermentation product. Appl Environ Microbiol 2004:70:5810-17.

47. Collado MC, Sanz Y. Method for direct selection of potentially probiotic Bifidobacterium strains from human feces based on their acid-adaptation ability. J Microbiol Methods 2006:66:560-3.

48. Kerckhoffs AP, Samsom M, van der Rest ME, et al. Lower Bifidobacteria counts in both duodenal mucosa-associated and fecal microbiota in irritable bowel syndrome patients. World J Gastroenterol 2009;15:2887-92.

49. Peterson DA, Frank DN, Pace NR, et al. Metagenomic approaches for defining the pathogenesis of inflammatory bowel diseases. Cell Host Microbe 2008;3:417-27.

50. Round JL, Mazmanian SK. Inducible Foxp3 + regulatory T-cell development by a commensal bacterium of the intestinal microbiota. Proc Natl Acad Sci USA 2010:107:12204-9.

51. Jernberg C, Löfmark S, Edlund C, et al. Long-term impacts of antibiotic exposure on the human intestinal microbiota. Microbiology (Reading, Engl) 2010;156:3216-23.

52. Kajander K, Krogius-Kurikka L, Rinttilä $T$, et al. Effects of multispecies probiotic supplementation on intestinal microbiota in irritable bowel syndrome. Aliment Pharmacol Ther 2007;26:463-73.

53. Charbonneau D, Baria M, Poehner R, et al. Impact of Bifidobacterium infantis 35624 on fecal flora from healthy and IBS subjects in a chemostat model. Gastroenterology 2001;128:A-661.

\section{DIFFERENTIAL DIAGNOSIS}

\section{Trustworthy guidance on your iPhone}
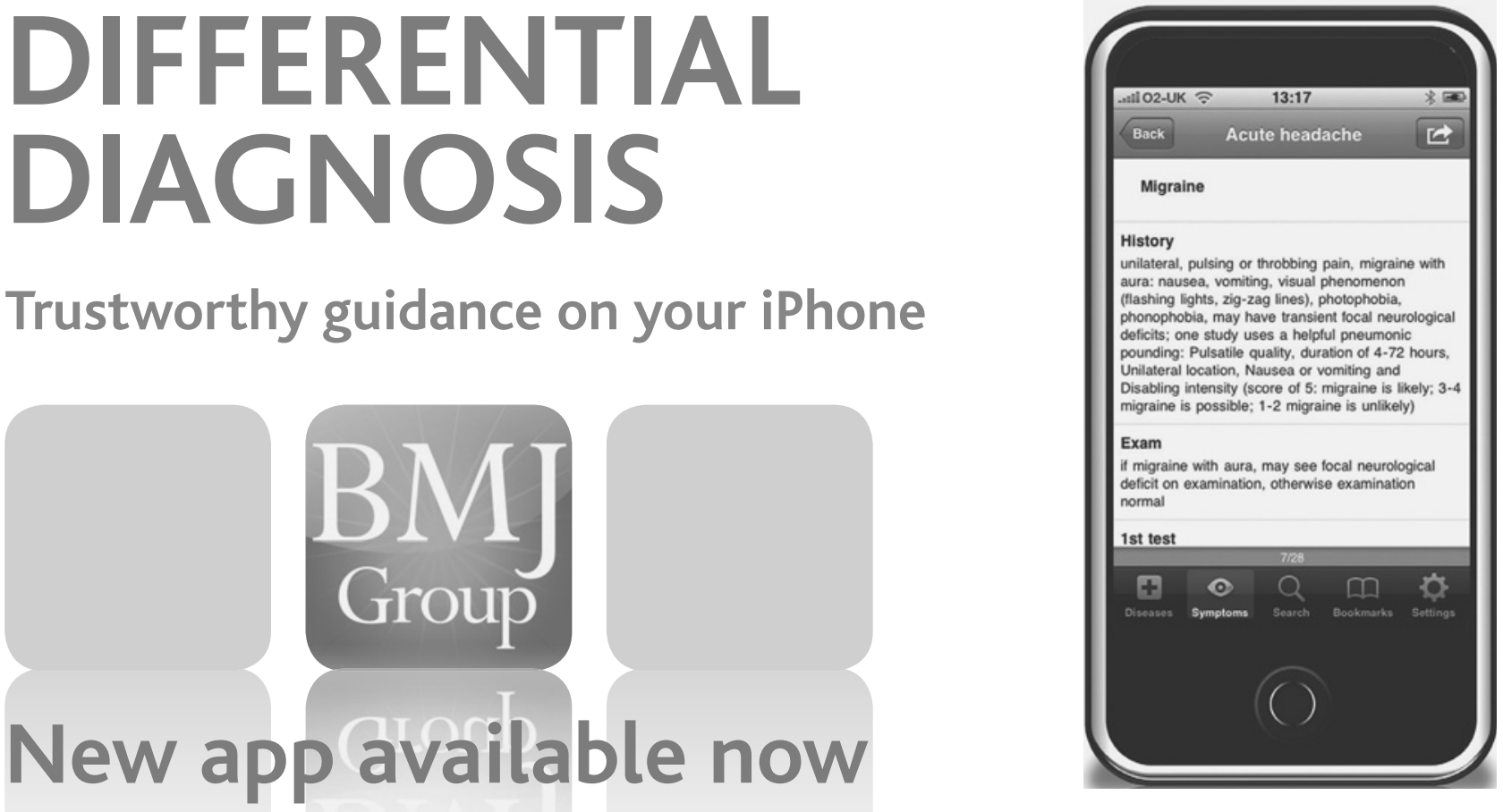

Find out more at bestpractice.bmj.com/differentials 


\section{GUT An irritable bowel syndrome subtype defined by species-specific alterations in faecal microbiota}

Ian B Jeffery, Paul W O'Toole, Lena Öhman, Marcus J Claesson, Jennifer

Deane, Eamonn M M Quigley and Magnus Simrén

Gut 2012 61: 997-1006 originally published online December 16, 2011

doi: 10.1136/gutjnl-2011-301501

Updated information and services can be found at:

http://gut.bmj.com/content/61/7/997

These include:

Supplementary Supplementary material can be found at:

Material http://gut.bmj.com/content/suppl/2011/12/16/gutjnl-2011-301501.DC1

References This article cites 50 articles, 9 of which you can access for free at: http://gut.bmj.com/content/61/7/997\#BIBL

Email alerting service

Receive free email alerts when new articles cite this article. Sign up in the box at the top right corner of the online article.

Topic Articles on similar topics can be found in the following collections

Collections

Irritable bowel syndrome (327)

\section{Notes}

To request permissions go to:

http://group.bmj.com/group/rights-licensing/permissions

To order reprints go to:

http://journals.bmj.com/cgi/reprintform

To subscribe to BMJ go to:

http://group.bmj.com/subscribe/ 